\title{
Multilayered composite structure design optimisation using distributed/parallel multi-objective evolutionary algorithms
}

\author{
D.S. Lee ${ }^{\mathrm{a}, *}$, C. Morillo ${ }^{\mathrm{b}}, \mathrm{G}$. Bugeda ${ }^{\mathrm{a}, \mathrm{b}}, \mathrm{S}$. Oller ${ }^{\mathrm{a}, \mathrm{b}}, \mathrm{E}$. Onate $\mathrm{O}^{\mathrm{a}, \mathrm{b}}$ \\ a International Center for Numerical Methods in Engineering (CIMNE), 08034 Barcelona, Spain ${ }^{1}$ \\ ${ }^{\mathrm{b}}$ University Politécnica de Cataluña, 08034 Barcelona, Spain
}

\section{A R T I C L E I N F O}

Article history:

Available online 17 October 2011

\section{Keywords:}

Multilayered composite structure

Fibre orientation

Stacking sequence

Multi-objective/multidisciplinary design

Distributed optimisation

Evolutionary algorithms

\begin{abstract}
A B S T R A C T
This paper presents a research work on stacking sequence design optimisation for multilayered composite plate using a parallel/distributed evolutionary algorithm. The stacking sequence of fibres has a dramatic influence on the strength of multilayered composite plates. Multiple layers of fibre-reinforced material systems offer versatility in engineering material design due to the fact that the stacking sequence of each orthotropic layer can offer full advantage of superior mechanical properties. Numerical results show that the optimal composite structures have lower weight, higher stiffness and also affordable cost when compared to the extreme and intermediate composite structures. In addition, the benefits of using a parallel optimisation system are also presented.
\end{abstract}

(c) 2011 Elsevier Ltd. All rights reserved.

\section{Introduction}

Multi-laminated composite structures are an ever-increasingly important topic in the fields of fabrication of mechanical, aerospace, marine, and machine industries due to their advantages such as durability (no corrosion - lower maintenance cost), survivability (fire resistance, crash energy absorption), excellent resistance against cyclic loading (no fatigue), reparability (restoration and repair), etc. [1,2]. Multilayered fibre-reinforced material systems can offer versatility in composite design due to the fact that the stacking sequence of each orthotropic layer can take full advantage of the superior mechanical properties in terms of its strength, stiffness, and total weight. One of the goals in design optimisation for multilayered composite structure is to increase its strength while lowering its weight with a given set of fibrous materials [2-5].

This paper presents a research work on stacking sequence design optimisation for multilayered composite structure in a discretised multi-objective approach using a parallel Multi-Objective Genetic Algorithm (MOGA) [6-8]. For the stacking sequence design variables, type of fibre, thickness and the orientation of fibres are considered for each layer of multilayered composite laminates. A combination of fibre types, thickness and orientation angles of each layer is computed by using a Finite Element Analysis (FEA) based composite structure analysis tool (COMPack) [9] to calculate the stiffness and strength parameters of a fibre-reinforced layer,

\footnotetext{
* Corresponding author.

E-mail addresses: dslee@cimne.upc.edu (D.S. Lee), cmorillo@cimne.upc.edu (C. Morillo), bugeda@cimne.upc.edu (G. Bugeda), sergio.oller@upc.edu (S. Oller), onate@cimne.upc.edu (E. Onate).

1 http://www.cimne.com
}

and also the total weight of laminates. For the optimisation, a MOGA implemented in a Robust Multi-objective Optimisation Platform (RMOP) developed in CIMNE is used under the parallel/distributed optimisation system and it is coupled to COMPack to find the optimal combination of stacking sequences for multilayered composite plates which have lower weight, higher stiffness and affordable total cost.

The paper is organised as follows; the description of the methodology is given in Section 2. Section 3 describes the analysis tool for composite structure. Section 4 conducts the multi-objective design optimisation for multilayered composite structure. Section 5 concludes the overall numerical results and shows the directions for future research avenue.

\section{Methodology}

\subsection{Multi-objective optimisation}

Often, engineering design problems require a simultaneous optimisation of conflicting objectives and an associated number of constraints. Unlike single objective optimisation problems, the solution is a set of points known as Pareto optimal set. Solutions are compared to other solutions using the concept of Pareto dominance. A multi-criteria optimisation problem can be formulated as:

Maximise/Minimise

$f_{i}(x)_{i} \quad i=1, \ldots, N$

Subject to constraints :

$g_{j}(x)=0 \quad j=1, \ldots, M$

$h_{k}(x) \leqslant 0 \quad k=1, \ldots, M$ 
where $f_{i}, g_{j}, h_{k}$ are, respectively, the objective functions, the equality and the inequality constraints. $N$ is the number of objective functions and $x$ is an $n$ - dimensional vector where its arguments are the decision variables. For a minimisation problem, a vector $x_{1}$ is said partially less than vector $x_{2}$ if:

$\forall_{i} f_{i}\left(x_{1}\right) \leqslant f_{i}\left(x_{2}\right)$ and $\exists_{i} f_{i}\left(x_{1}\right) \leqslant f_{i}\left(x_{2}\right)$

In this case the solution $x_{1}$ dominates the solution $x_{2}$.

As Genetic Algorithms (GAs) evaluate multiple populations of points, they are capable of finding a number of solutions in a Pareto set. Pareto selection ranks the population and selects the non-dominated individuals for the Pareto fronts. A Genetic Algorithm that has capabilities for multi-objective optimisation is termed MultiObjective Genetic Algorithms (MOGAs). Theory and applications of MOGAs can be found in Refs. [6-8].

\subsection{Robust Multi-objective Optimisation Platform (RMOP)}

RMOP is a computational intelligence framework which is a collection of population based algorithms including Genetic

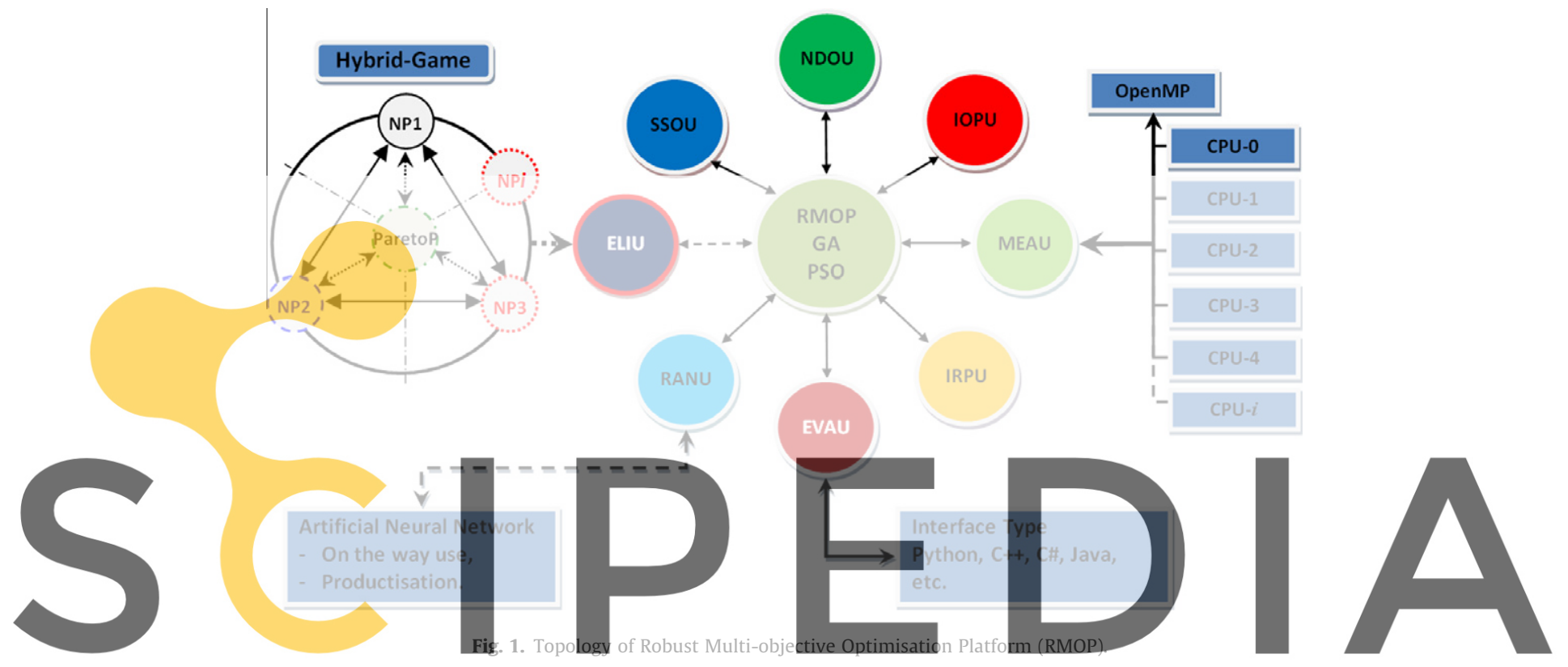

Register for free at https//www.scipedia.com to download the version without the watermark

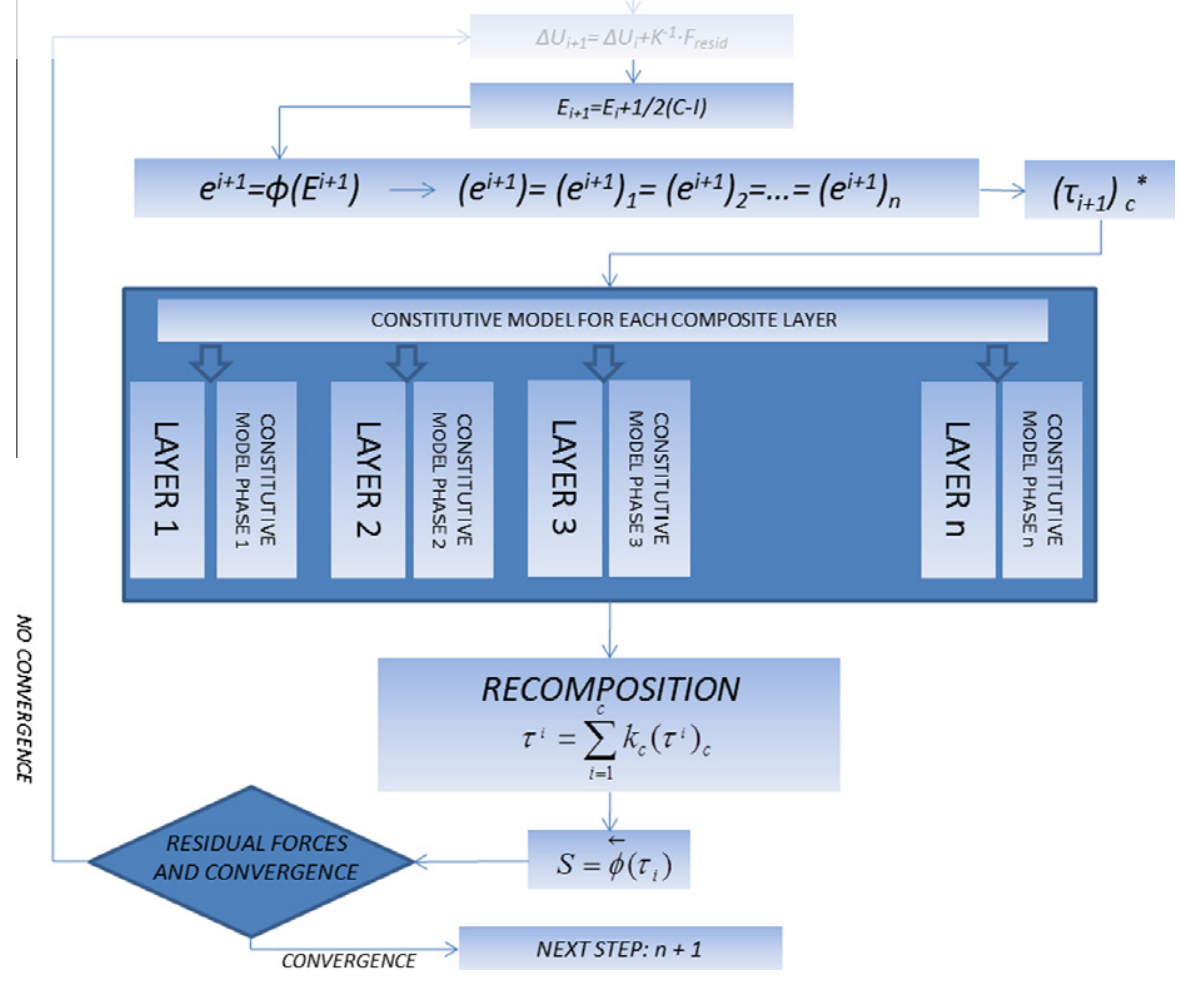

Fig. 2. Mechanism of COMPack. 

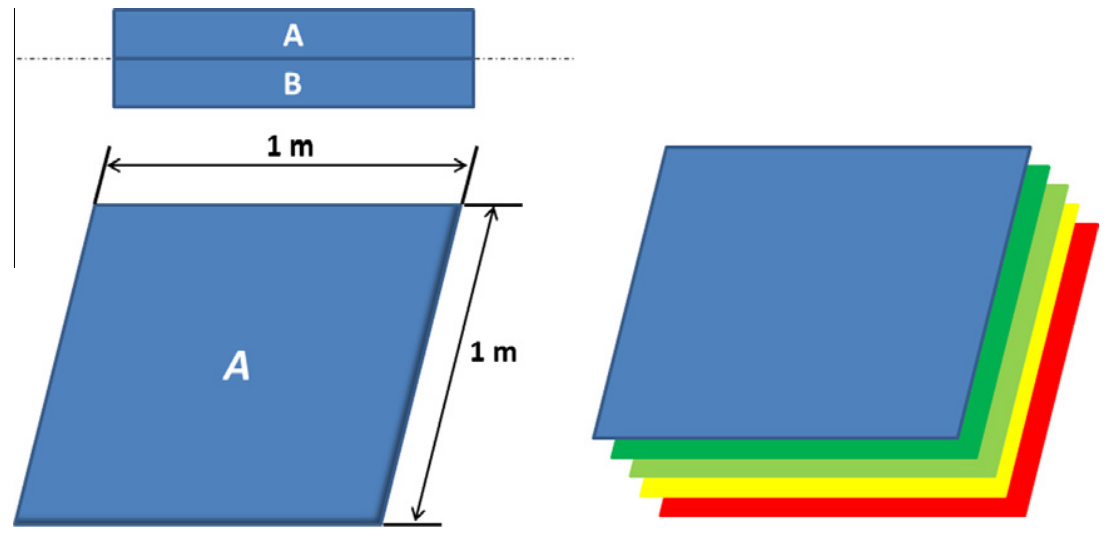

Fig. 3. Baseline multilayered composite design.

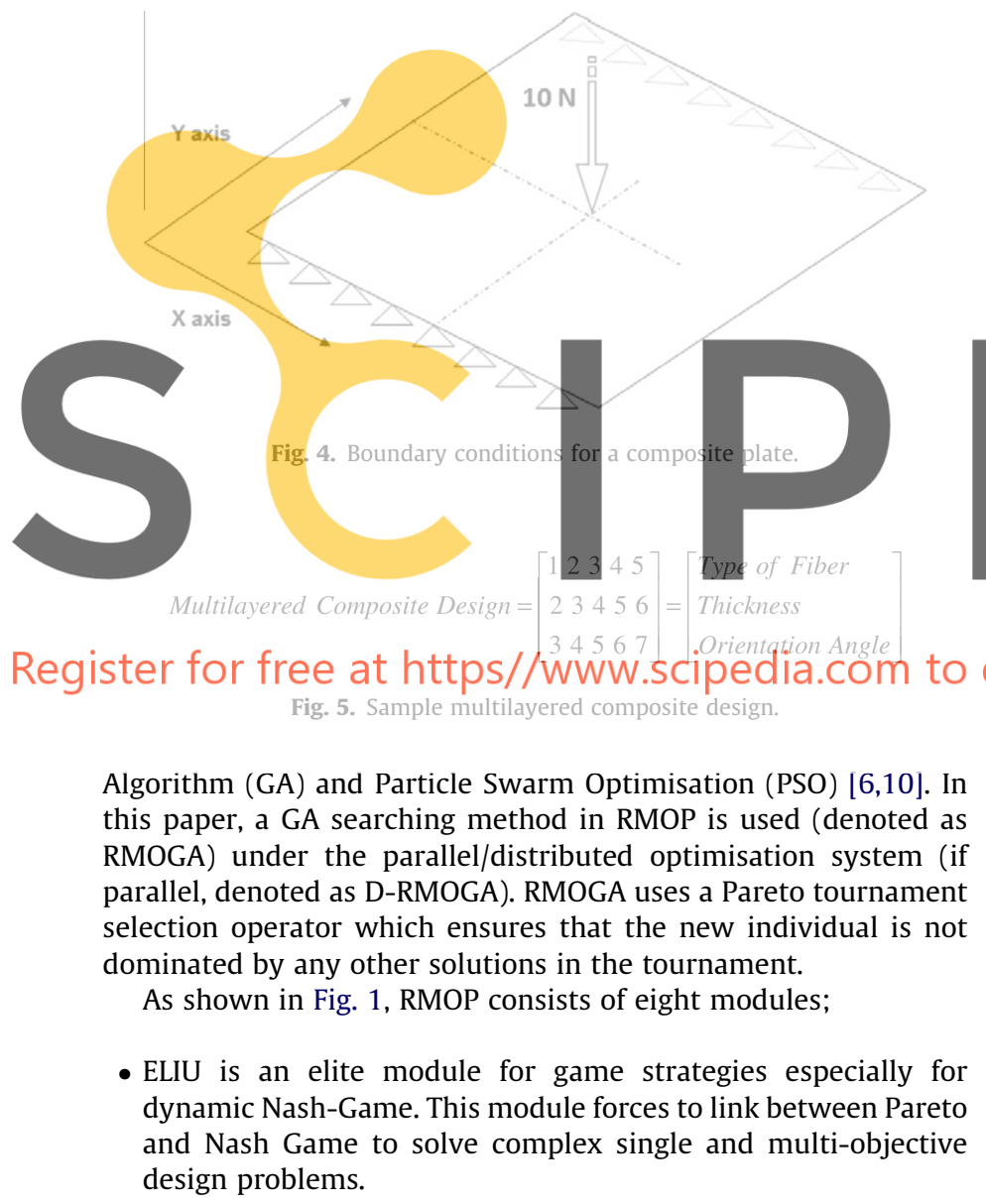

Table 3

Fibre orientation angles.

\begin{tabular}{ccccccccccccc}
\hline ID \# & 1 & 2 & 3 & 4 & 5 & 6 & 7 & 8 & 9 & 10 & 11 & 12 \\
\hline $\begin{array}{r}\text { Angle } \\
\left({ }^{\circ}\right)\end{array}$ & 0 & 15 & 30 & 45 & 60 & 75 & 90 & -15 & -30 & -45 & -60 & -75
\end{tabular}

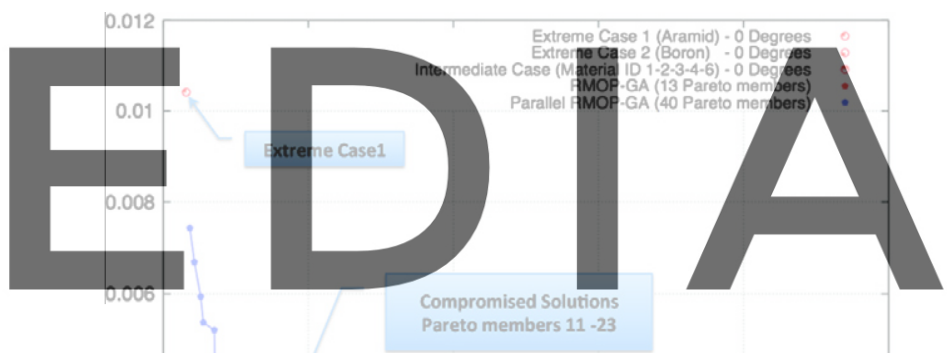

dowadoad the version without the watermark

Algorithm (GA) and Particle Swarm Optimisation (PSO) [6,10]. In this paper, a GA searching method in RMOP is used (denoted as RMOGA) under the parallel/distributed optimisation system (if parallel, denoted as D-RMOGA). RMOGA uses a Pareto tournament dominated by any other solutions in the tournament.

As shown in Fig. 1, RMOP consists of eight modules;

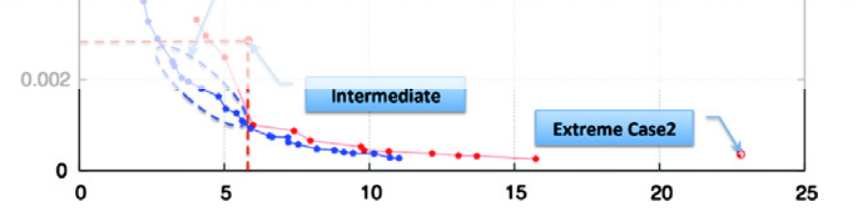

Fig. 6. Pareto optimal front.

- EVAU is a module for evaluation and collecting results from analysis tools. It is also capable to handle other language-based interfaces.

- IOPU is a module for handling input, output data and also plotting convergence history, initial population (with/without buffer population), total populations, Pareto optimal front.

Table 1

Generic fibre material types. (Note: The density for matrix (epoxy) is $1.800 \mathrm{e}+3$ and $40 \%$ of this will be applied to each layer.)

\begin{tabular}{lllllll}
\hline ID \# & 1 & 2 & 3 & 4 & 5 & 6 \\
\hline Name & Carbon Pan & Carbon Pitch & Carbon Rayon & Glass & Aramid \\
Density $\left(\mathrm{kg} / \mathrm{m}^{3}\right)$ & $1.825 \mathrm{e}+3$ & $2.025 \mathrm{e}+3$ & $1.600 \mathrm{e}+3$ & $2.550 \mathrm{e}+3$ & $1.440 \mathrm{e}+3$ & Boron \\
Cost $(\mathrm{US} \$ / \mathrm{kg})$ & 60 & 120 & 15 & 22.5 & 50 & $175 \mathrm{e}+3$ \\
\hline
\end{tabular}

Table 2

Layer thicknesses (m).

\begin{tabular}{|c|c|c|c|c|c|c|c|c|c|c|c|}
\hline ID \# & 1 & 2 & 3 & 4 & 5 & 6 & 7 & 8 & 9 & 10 & 11 \\
\hline Thickness & $1 \times 10^{-4}$ & $2 \times 10^{-4}$ & $4 \times 10^{-4}$ & $6 \times 10^{-4}$ & $8 \times 10^{-4}$ & $1 \times 10^{-3}$ & $1.2 \times 10^{-3}$ & $1.4 \times 10^{-3}$ & $1.6 \times 10^{-3}$ & $1.8 \times 10^{-3}$ & $2 \times 10^{-3}$ \\
\hline
\end{tabular}


Table 4

Comparison of weight and displacement obtained by the baseline (extreme and intermediate cases) composite and the multi-objective optimal multilayered composites.

\begin{tabular}{lll}
\hline Type of composite & Weight $(\mathrm{kg})$ & $\begin{array}{l}\text { Displacement } \\
(\mathrm{m})\end{array}$ \\
\hline Extreme Case 1 (lighter) & 0.792 & 0.01042 \\
Pareto member 1 & $0.919(+16.0 \%)$ & $0.00744(-28.6 \%)$ \\
Intermediate composite & 5.558 & 0.00286 \\
Pareto member 15 & $3.283(-41.0 \%)$ & $0.00194(-32.2 \%)$ \\
$\quad$ (compromised solution) & & \\
Extreme Case 2 (heavier) & 22.8 & 0.00036 \\
Pareto member 40 & $11.023(-51.6 \%)$ & $0.00026(-28.0 \%)$
\end{tabular}

- IRPU is an initial random population module.

- MEAU is a module for allocating/dis-allocating memory for population and it provide Parallel/Distributed optimisation environment

- NDOU is a module for computing Pareto-tournament, non-dominated sorting solutions from population.

- RANU is a module for generating pseudo random number module and Artificial Neural Network (ANN).

- SSOU is a searching module; selection, mutation, crossover for GA and also it produces velocity, positioning module for PSO.

RMOP is easily coupled to any analysis tools such as Computa-
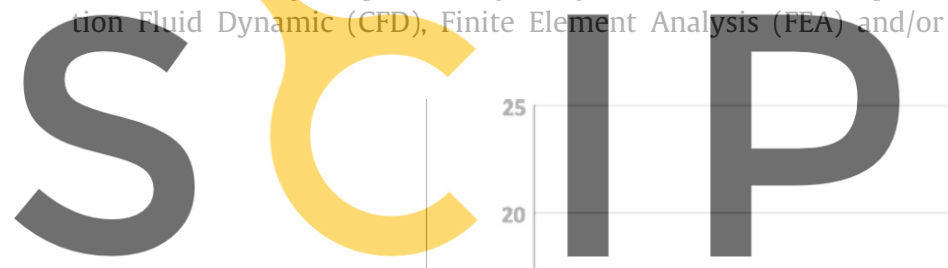

Computer Aided Design (CAD) systems. In addition, it is capable to solve any engineering design application $[11,12]$.

\section{Analysis of laminated composite structures}

\subsection{Analysis of laminated composite structure}

The analysis of composite structure by FEM based on the classical mixture theory [13] follows the algorithm shown in Fig. 2, in non-linear problems with finite deformations for a multi-phase composite material.

Each of those phases corresponds to a layer of the composite plate and it has its own constitutive model independent of the other phases. The algorithm starts in the reference configuration and then, by means of stress transport operations, from the referential to updated configuration ("push-forward") resolves the constitutive equation for each of the phases that form the composite material $[9,14-16]$. Each of those phases can be isotropic or anisotropic and can present a different type of constitutive behaviour. Once determined the stress state of each component it's needed to find the total stress of the composite material by Eq. (1), it allows also finding the internal forces in each point of the structure,

$$
\sigma_{i j}=C_{i j k l}^{S} \varepsilon_{k l}^{e}=\sum_{c=1}^{n} k_{c}\left(\sigma_{i j}\right)_{c}=\sum_{c=1}^{n} k_{c}\left(C_{i j k l}^{S} \varepsilon_{k l}^{e}\right)_{c}
$$

where $\varepsilon_{k l}^{e}$ is the strain tensor for the composite and $k_{c}$ represents the volumetric participation of component $c$ in the composite i.e.
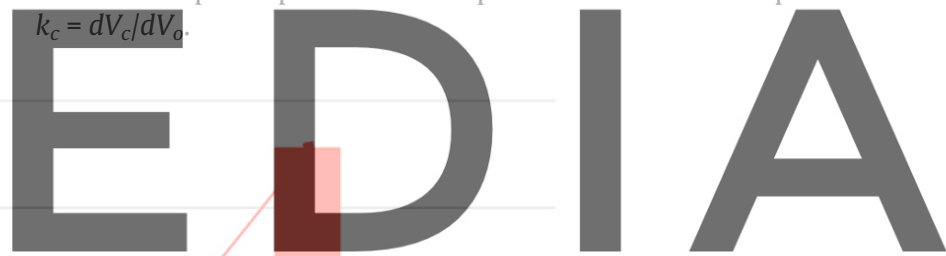

Register for free at htt露s//www.scipedia.com to download the version without the watermark
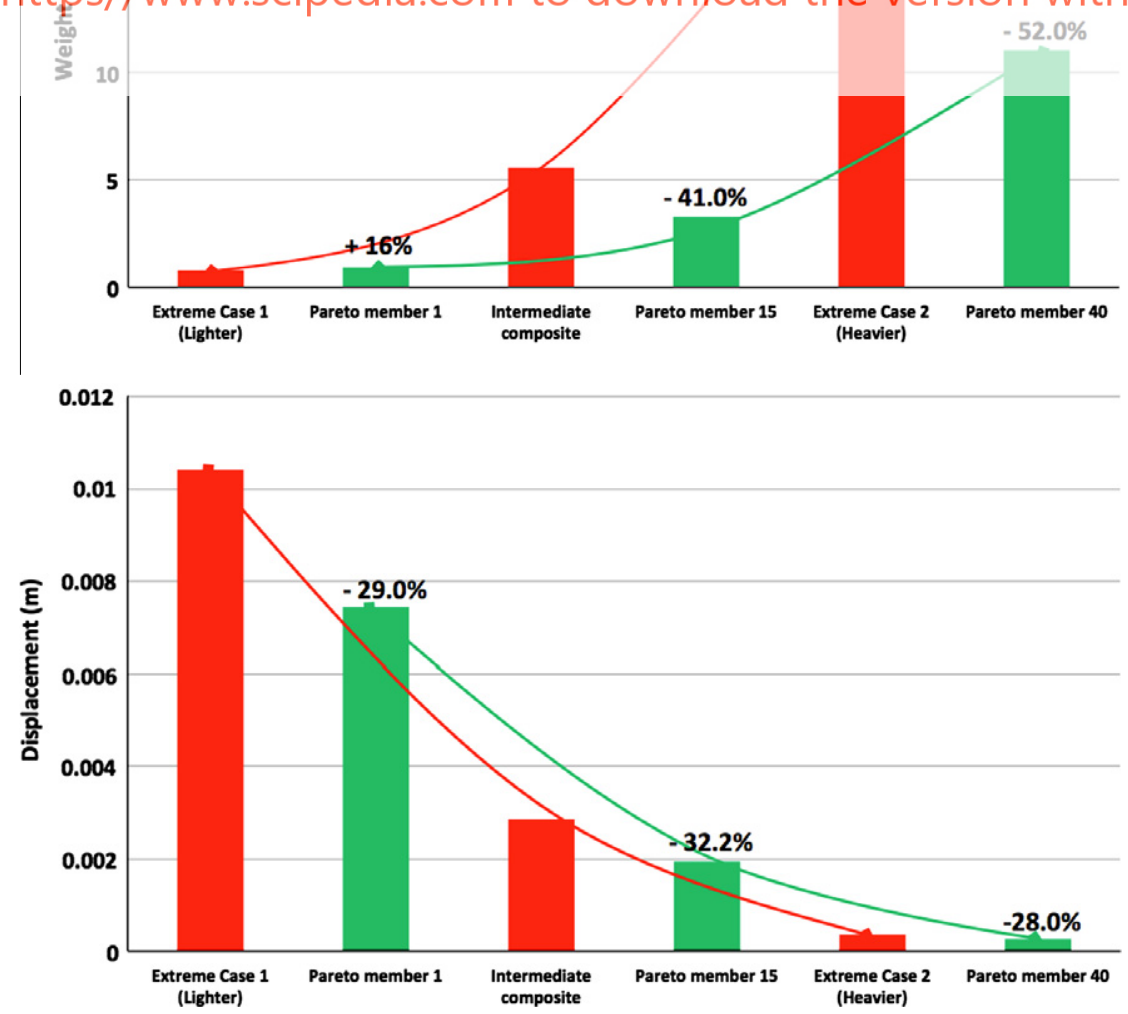

Fig. 7. Histogram for weight (top) and displacement (bottom) obtained by the extreme, intermediate cases and Pareto members 1,15 and 40 obtained by D-RMOGA. 
Later, it's possible to verify the balance between those forces and the external applied forces. Details of this theory including extension to the large deformation cases and its numerical implementation can be found in Ref. [15].

\subsection{Analysis tools (COMPack)}

COMPack in an analysis kit designed by Quantech and CIMNE able to use the necessary tools in order to create the finite element model to perform structural simulations of composite material structures [8,14-16]. COMPack is able to determine the structural properties such as elastic behaviour, ultimate tensile and compression strength and damage level of a composite material. One of the principal benefits of COMPack it's the capability of working with the constitutive model of the composite material in detail. To do so, it takes in account all mechanical and physical properties, amount and orientation of each of its forming fibre and matrix materials, and follows a FEM procedure to solve the structural problem.

\section{Stacking sequence design optimisation for multilayered composite structure \\ 4.1. Problem formulation}

The problem considers a multi-objective composite stacking sequence design optimisation to find lighter and stiffer multilayered composite structures. Fig. 3 shows the baseline composite consisting of symmetric (non-balanced) lami laminate $A$ has five layers; each lay of epoxy. The boundary conditions shown in Fig. 4 where a quadrilate
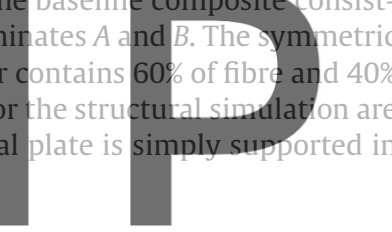

Table 5

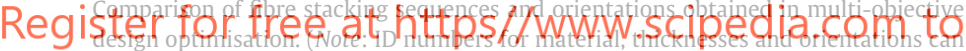
be found in Tables 1-3.)

\begin{tabular}{llll}
\hline Composite & $\begin{array}{l}\text { Stacking } \\
\text { sequence }\end{array}$ & Thicknesses & Orientations \\
\hline Extreme Case 1 & $5-5-5-5-5$ & $1-1-1-1-1(0.0005 \mathrm{~m})$ & $1-1-1-1-1$ \\
Pareto member 1 & $5-1-6-2-5$ & $1-1-1-1-1(0.0005 \mathrm{~m})$ & $4-6-4-7-4$ \\
Intermediate Case & $2-3-4-5-6$ & $4-4-4-4-4(0.003 \mathrm{~m})$ & $1-1-1-1-1$ \\
Pareto member 15 & $1-3-1-1-5$ & $1-2-2-5-4(0.0019 \mathrm{~m})$ & $3-3-7-5-4$ \\
Extreme Case 2 & $6-6-6-6-6$ & $11-11-11-11-11(0.01 \mathrm{~m})$ & $1-1-1-1-1$ \\
Pareto member 40 & $1-5-3-5-1$ & $9-9-9-8-2(0.0082 \mathrm{~m})$ & $5-11-2-4-1$ \\
\hline
\end{tabular}

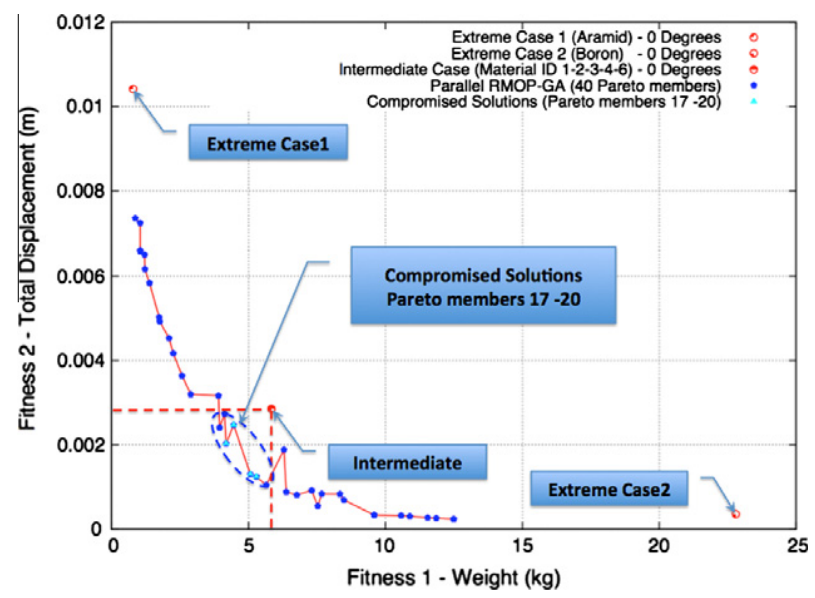

Fig. 8. Fitness 2; displacement (m) vs. Fitness 1; weight (kg). two of its sides and a constant punctual force is applied in the central position.

\subsection{Design variables}

The candidate multilayered composite consists of five layers with five fibrous materials, five thicknesses and five orientation angles, as shown in Fig. 5. Design variables are limited to six orthotropic materials, eleven thicknesses, and twelve orientation angles as shown in Tables 1-3. It can be predicted that the lighter multi-
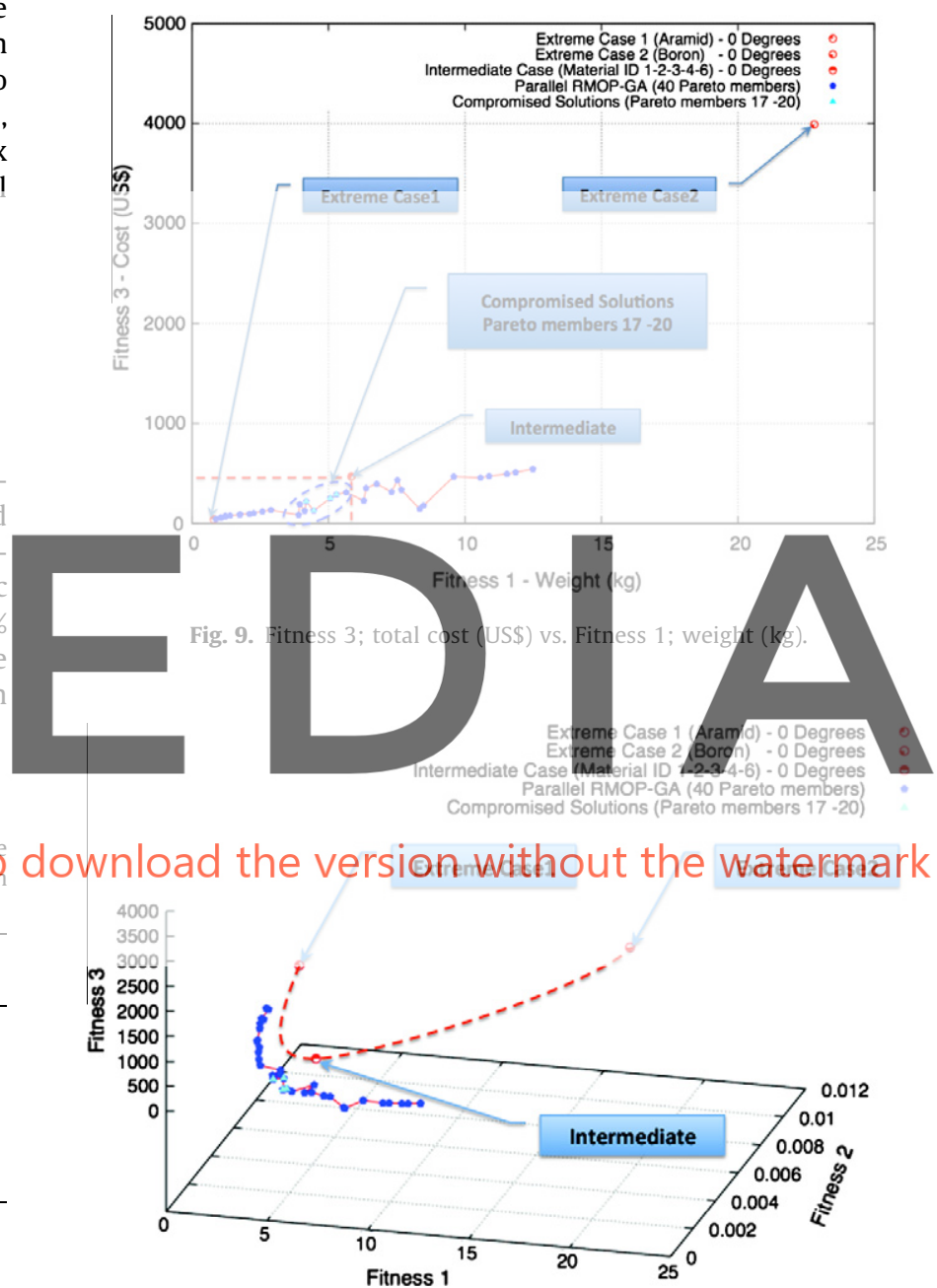

Fig. 10. 3D view of Pareto front obtained by D-RMOGA.

Table 6

Comparison of multilayered composite weight, displacement, and total cost obtained by the baseline (extreme and intermediate cases) composite and the multidisciplinary optimal multilayered composites.

\begin{tabular}{llll}
\hline Type of composite & $\begin{array}{l}\text { Weight } \\
(\mathrm{kg})\end{array}$ & $\begin{array}{l}\text { Displacement } \\
(\mathrm{m})\end{array}$ & $\begin{array}{l}\text { Total cost } \\
(\mathrm{US} \$)\end{array}$ \\
\hline Extreme Case 1 (lighter) & 0.792 & 0.01042 & 39.6 \\
Pareto member 1 & 0.870 & 0.00735 & $43.1(+8.8 \%)$ \\
& $(+9.8 \%)$ & $(-29.5 \%)$ & \\
Intermediate composite & 5.558 & 0.00286 & 471.74 \\
Pareto member 17 & 4.184 & 0.00203 & 221.85 \\
$\quad$ (compromised solution) & $(-25.0 \%)$ & $(-29.0 \%)$ & $(-53.0 \%)$ \\
Extreme Case 2 (heavier) & 22.8 & 0.00036 & 3990.0 \\
Pareto member 40 & 12.489 & 0.00023 & 542.16 \\
& $(-45.2 \%)$ & $(-36.1 \%)$ & $(-86.4 \%)$ \\
\hline
\end{tabular}


layered composite will be a combination of five Aramid (Material ID - 5) with lower thickness (Thickness ID - 1) and $0^{\circ}$ orientation angles, denoted as Extreme Case 1, while the heaviest multilayered composite will be a combination of five Boron (Material ID - 6) with higher thickness (Thickness ID - 11) and $0^{\circ}$ orientation angles, denoted as Extreme Case 2. One intermediate multilayered composite can be a combination of Material ID (1-2-3-4-6) with thickness ID $-4(0.6 \mathrm{~mm})$ and $0^{\circ}$ orientation angles. The details of orthotropic material can be found in Ref. [17].

\subsection{Multi-objective multilayered composite structure design optimisation}

\subsubsection{Problem definition}

The problem considers a multi-objective multilayered composite structure design optimisation using D-RMOGA (with parallel) and RMOGA (without parallel). The fitness functions are to minimise the weight of the multilayered composite while minimising its maximum displacement as shown in the following equations:

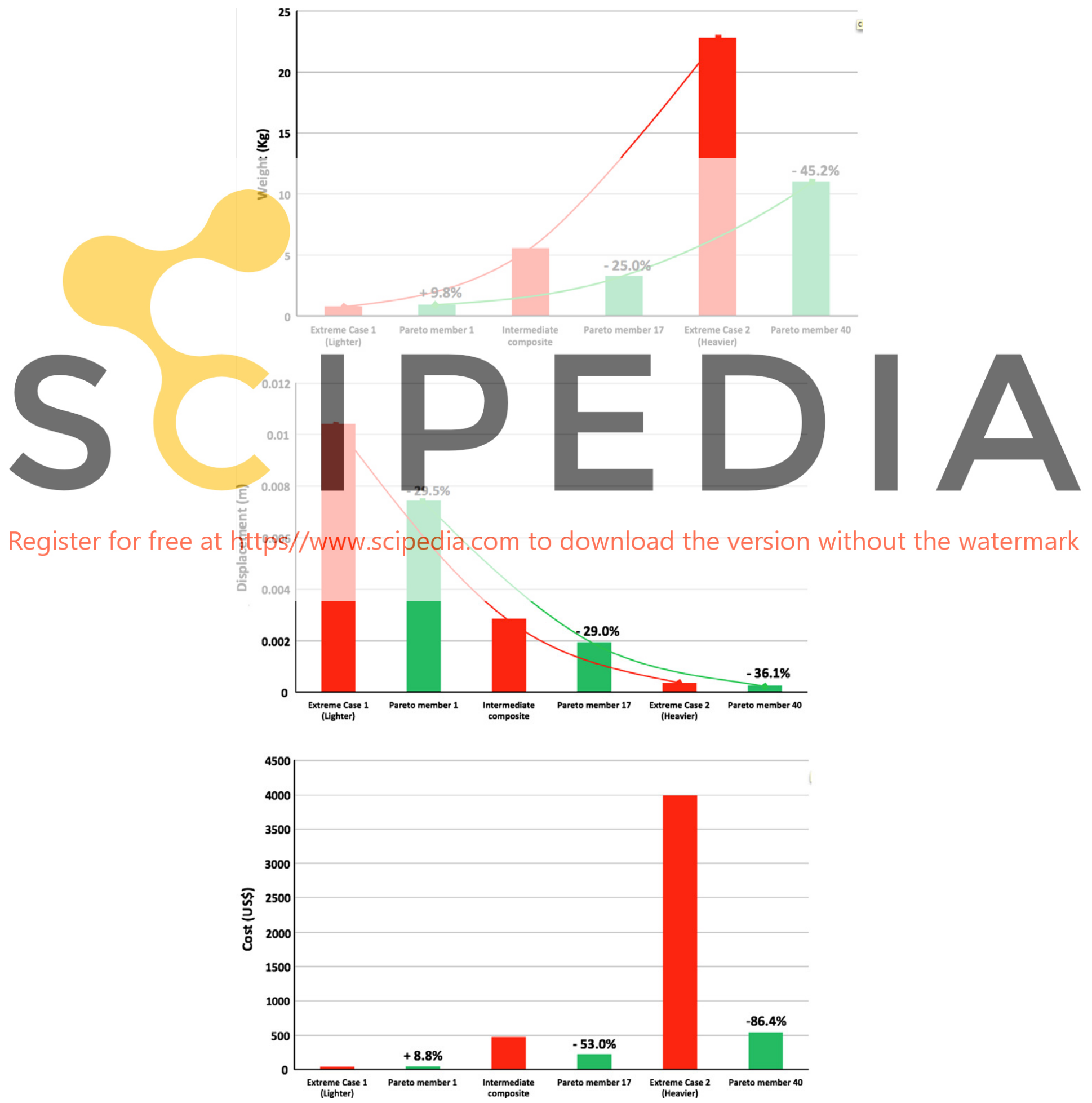

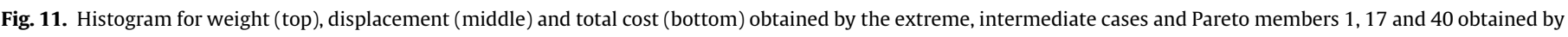
D-RMOGA. 
Table 7

Comparison of fibre stacking sequences and orientations obtained in multidisciplinary design optimisation. (Note: ID numbers for material, thicknesses and orientations can be found in Tables 1-3.)

\begin{tabular}{llll}
\hline Composite & $\begin{array}{l}\text { Stacking } \\
\text { sequence }\end{array}$ & Thicknesses & Orientations \\
\hline Extreme Case 1 & $5-5-5-5-5$ & $1-1-1-1-1(0.0005 \mathrm{~m})$ & $1-1-1-1-1$ \\
Pareto member 1 & $1-1-5-1-3$ & $1-1-1-1-1(0.0005 \mathrm{~m})$ & $11-1-9-5-2$ \\
Intermediate Case & $2-3-4-5-6$ & $4-4-4-4-4(0.003 \mathrm{~m})$ & $1-1-1-1-1$ \\
Pareto member 17 & $1-3-1-5-2$ & $3-3-1-8-2(0.0025 \mathrm{~m})$ & $5-9-4-6-8$ \\
Extreme Case 2 & $6-6-6-6-6$ & $11-11-11-11-11(0.01 \mathrm{~m})$ & $1-1-1-1-1$ \\
Pareto member 40 & $5-3-5-5-5$ & $11-8-10-9-6(0.0078 \mathrm{~m})$ & $1-3-8-5-11$ \\
\hline
\end{tabular}

$f_{1}=\min \left(W_{\text {Composite }}\right)=\sum_{i=0}^{n} W_{\text {Layer }_{i}}$

$$
f_{2}=\min \left(d_{\text {Total }}\right)=\sqrt{\left(\Delta x^{2}+\Delta y^{2}+\Delta z^{2}\right)}
$$

where $W_{\text {Composite, }} d_{\text {Total }}$ represent the weight and displacement of the multilayered composite, respectively.

\subsubsection{Numerical results}

Two optimisation algorithms; D-RMOGA and RMOGA have run $10 \mathrm{~h}$ of computer time (760 and 120 function evaluations respectively). The number of processors usage for RMOGA and D-RMOGA are one and ten CPUs in Dell PowerEdge 6850 (Intel(R) Xeon(TM) CPU $16 \times 3.20 \mathrm{GHz}$ and $32 \mathrm{~GB}$ RAM) machine. Fig. 6 compares the Pareto optimal front obtained by the D-RMOGA and RMOGA with the two extreme cases (lightest multilayered composite and heaviest multilayered composite) and one intermediate case. It is clearly shown that the Pareto front obtained by D-RMOGA (with ten parallel CPUs) has a better convergence and divergence when compared to the Pareto front obtained by RMOGA. Pareto members 11-23 dominate the intermediate multilayered composite in terms of weight and displacement. Pareto members 1,15 and 40 are selected to compare to the extreme cases 1,2 and intermediate composites.

Table 4 compares the weight and displacement obtained by the extreme, intermediate cases and Pareto ontimal solutions (Pareto members 1,15 and 40). Pareto member 1 (the best optimal solution for weight) has $29 \%$ lower displacement when compared to the extreme case 1 even though it is $16 \%$ heavier. Pareto member
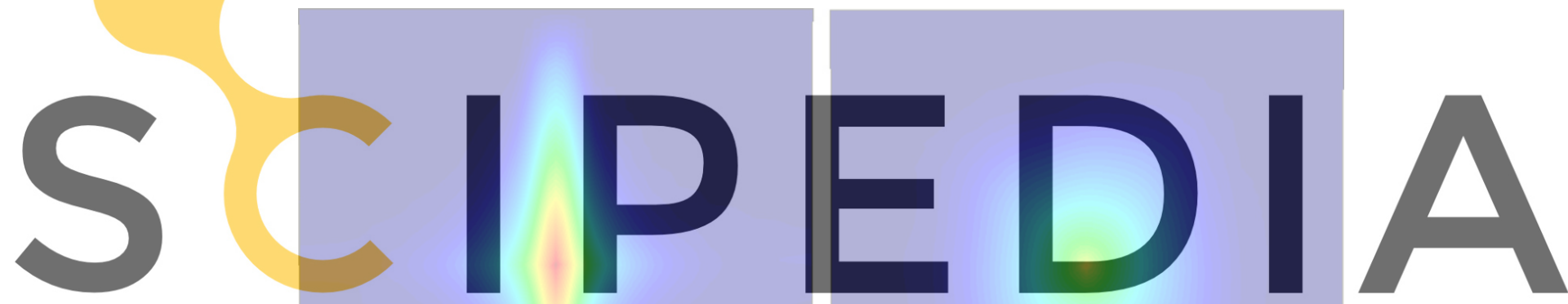

Register for free at https//www.scipedia.com to

download the version without the watermark
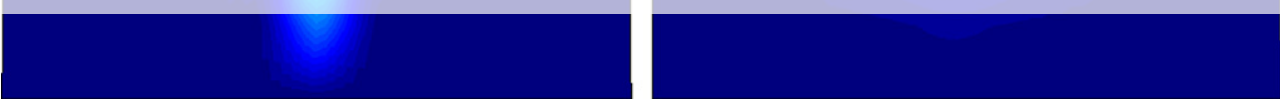

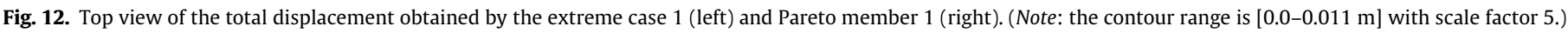
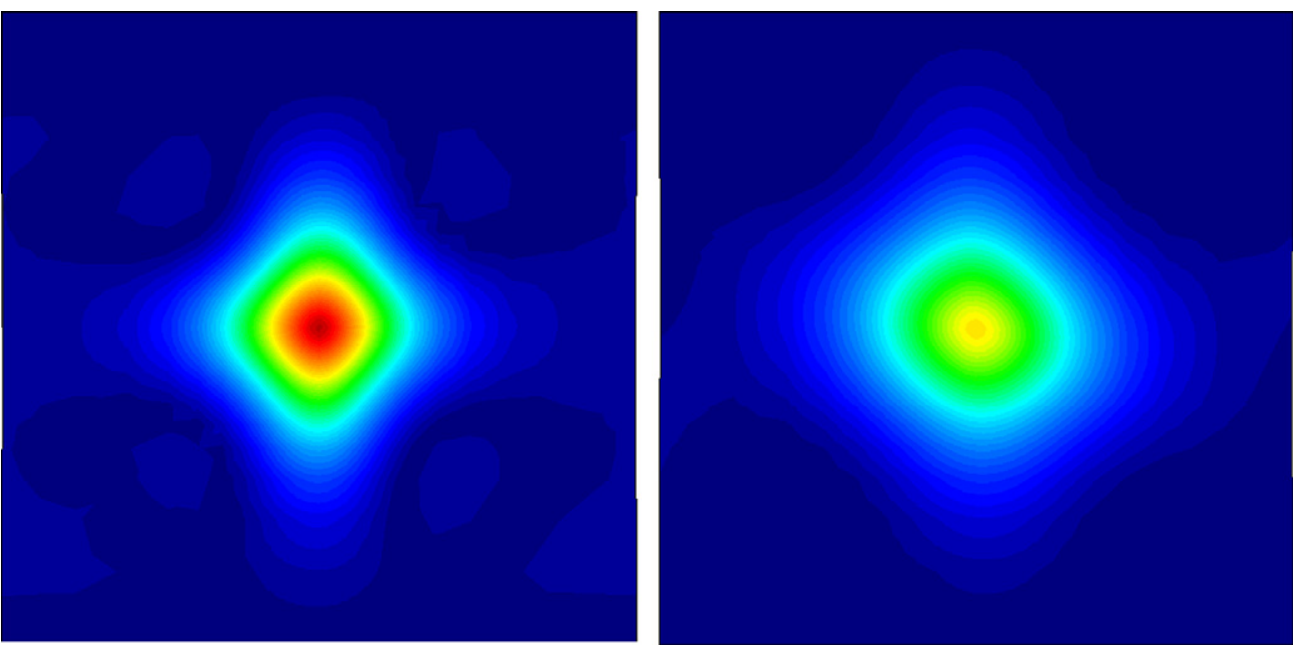

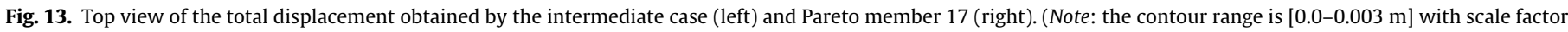
20.) 

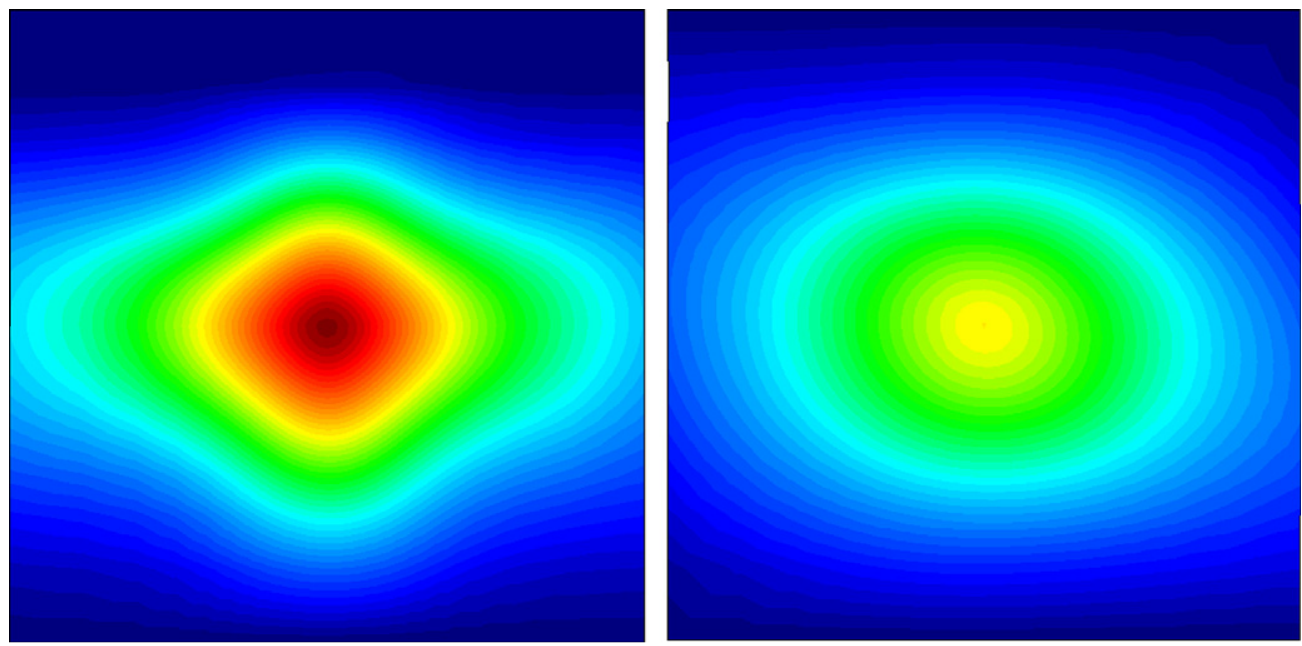

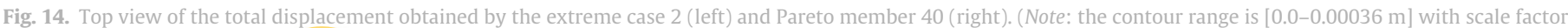
100.)
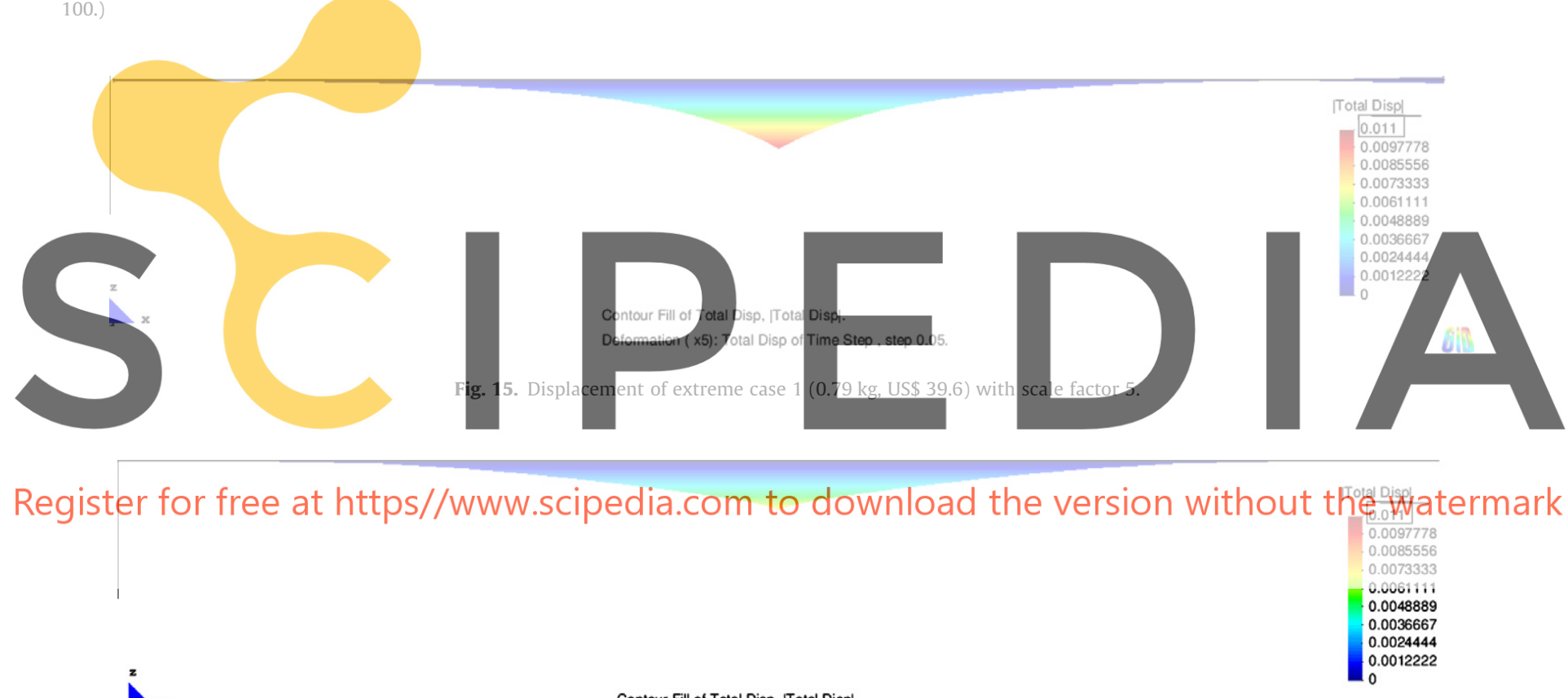

Contour Fill of Total Disp, |Total Displ.

Deformation ( $\times 5$ ): Total Disp of Time Step , step 0.05 .

Fig. 16. Displacement of Pareto member $1(0.87 \mathrm{~kg}$, US\$ 43.1$)$ with scale factor 5 .

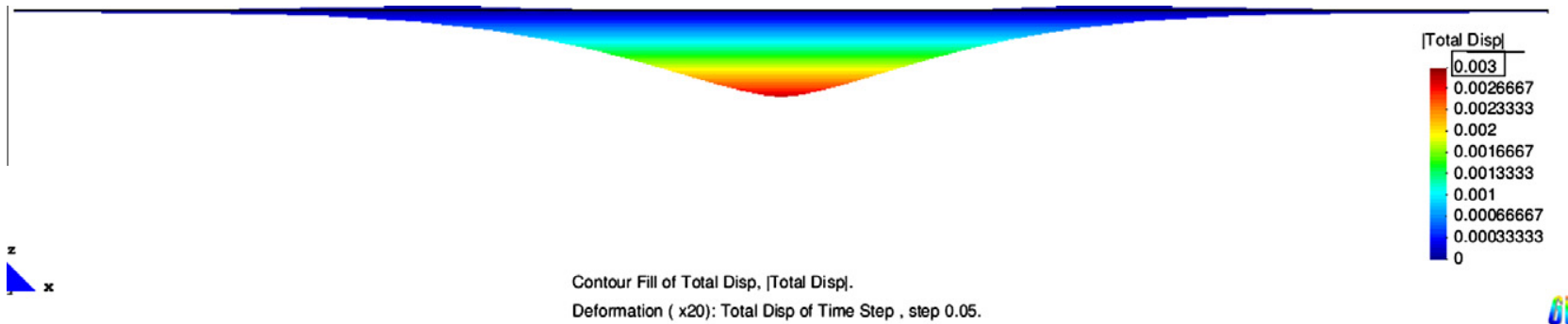

Fig. 17. Displacement of intermediate case $(5.56 \mathrm{~kg}$, US\$ 471.74) with scale factor 20.

40 (the best optimal solution for displacement) has $52 \%$ lower weight while having $28 \%$ lower displacement when compared to the extreme case 2. Pareto member 15 (one of compromised solutions for weight and displacement) has $41 \%$ and $32 \%$ lower weight and displacement when compared to the intermediate case. The histograms shown in Fig. 7 compare the weight and the displacement obtained by the extreme, the intermediate cases and the Pareto optimal solutions.

Table 5 compares the fibre stacking sequences and the layer orientations for the extreme cases, the intermediate and the Pareto 


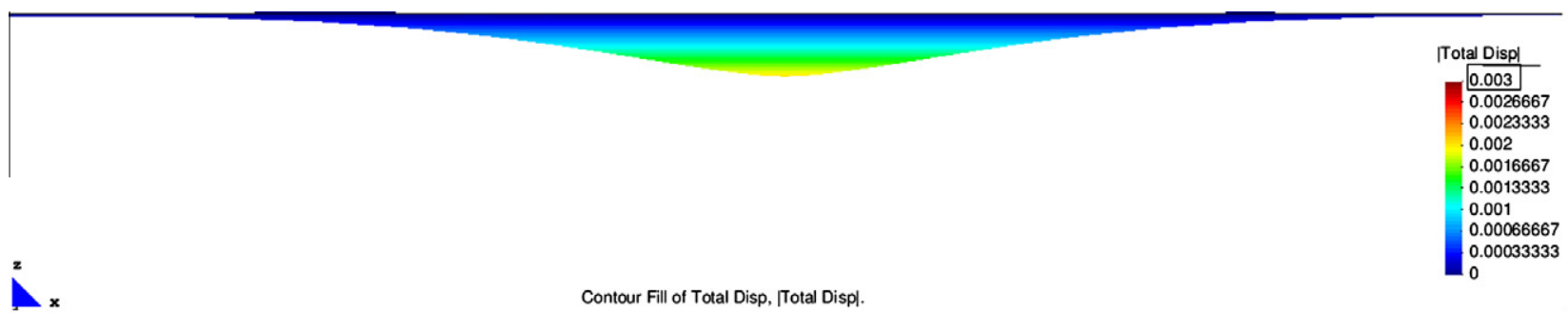

Deformation ( $\mathrm{x20}$ ): Total Disp of Time Step , step 0.05 .

Fig. 18. Displacement of Pareto member 17 (4.18 kg, US\$221.85) with scale factor 20

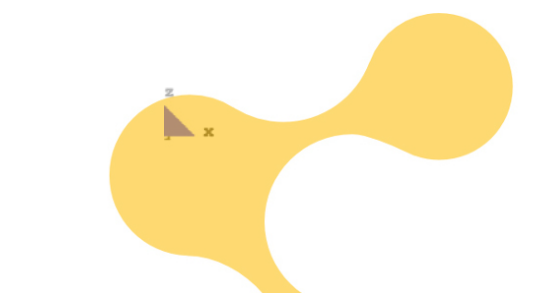

\section{Contour Fill of Total Disp, |Total Disp|.
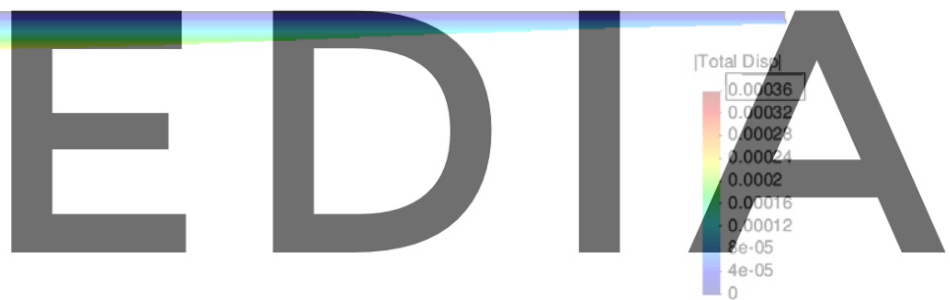

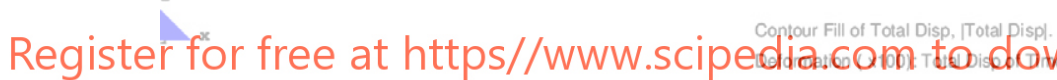

Fig. 20. Displaycement of Pareto member 40 (12.5 kg, US\$542.16) with scale factor 100.

optimal solutions. One fact that can be noticed is that the Pareto optimal solutions mainly use lower density fibres (Aramid and Carbon Pan). They have lower displacements even though the Pareto optimal solutions have lower total thickness when compared to the extreme cases and the intermediate composites. This is mainly due to appropriate material sequences and orientations.

\subsection{Multidisciplinary multilayered composite structure design optimisation}

\subsubsection{Problem definition}

The problem considers a multidisciplinary multilayered composite structure design optimisation using D-RMOGA with 10 CPUs. The fitness functions are to minimise both the weight and displacement of the multilayered composite while reducing the cost of composite structure as shown in Eqs. (2)-(4);

$f_{3}=\min \left(C_{\text {composite }}\right)=\sum_{i=0}^{n}\left(W_{\text {Layer }_{i}} \times P_{\text {Layer }_{i}}\right)$

where $C_{\text {Composite }}, W_{\text {Layer }}, P_{\text {Layer }}$ represent the total cost for the multilayered composite structure, the weight and price for each layer respectively.

\subsubsection{Numerical results}

The optimisation procedure has taken $20 \mathrm{~h}$ of computer time (1600 function evaluations). Figs. 8-10 show the Pareto optimal front obtained by the D-RMOGA compared with the two mentioned extreme cases (lightest multilayered composite and heaviest multilayered composite) and one intermediate case. It can be seen that Pareto members 14 to 20 dominate the intermediate multilayered composite. Pareto members 1,17 and 40 are selected to compare to the extreme cases 1,2 and intermediate composites.

Table 6 compares the weight, displacement and total cost obtained by the extreme, intermediate cases and Pareto optimal solutions (Pareto members 1, 17 and 40). Pareto member 1 (the best optimal solution for weight) has $30 \%$ lower displacement when compared to the extreme case 1 even though it is $10 \%$ heavier and $9 \%$ expensive. Pareto member 40 (the best optimal solution for displacement) has $45 \%$ lower weight while having 36\% lower displacement with $86 \%$ lower total cost when compared to the extreme case 2. Pareto member 17 (one of compromised solutions for weight, displacement and cost) has lower weight by $25 \%$, lower displacement by $29 \%$, and $53 \%$ lower total cost when compared to the intermediate case. The histograms shown in Fig. 11 compare the weight, displacement, total cost of composite structures obtained by the extreme, the intermediate cases and the Pareto optimal solutions.

The fibre stacking sequences and the layer orientations obtained by the extreme cases, the intermediate case and the Pareto optimal solutions are compared as shown in Table 7. It can be seen that the composite structures obtained by the Pareto optimal solutions mainly consist of lower density fibres; Aramid and Carbon 
Pan, which results in lower weight composite structure. Even though the Pareto optimal solutions have lower total thickness, they still have lower displacements and lower total cost when compared to the extreme and the intermediate composites. This is mainly due to appropriate material sequences and orientations.

Figs. 12-14 show the top view of the total displacement obtained by the extreme and intermediate cases, Pareto members 1 , 17 and 40. It can be seen that Pareto members 1, 17 and 40 have less displacement at the center when compared to the extreme and intermediate cases.

Figs. 15-20 show the side view ( $x$ and $z$-axis) of the displacement contours obtained by the extreme cases, intermediate and Pareto optimal solutions.

\section{Conclusion}

In this paper, a methodology for the stacking sequence design optimisation of multilayered composite structure has been described and investigated. The methodology couples a robust multi-objective evolutionary algorithm and a finite element analysis based composite structure analysis tool under a parallel optimisation system. It has been implemented to improve the composite structure in terms of both the mechanical properties (weight, and stiffness) and the industrial affordability (cost). Analytical research shows that Pareto optimal solutions obtained from the optimisation offers a set of selections to design engineers so that they may proceed into more detail phases of the composite structure

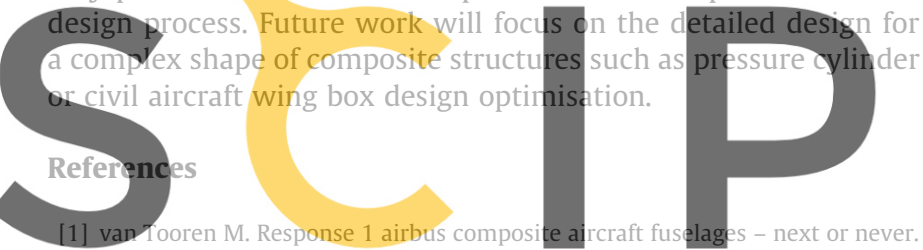
Around glare a new aircraft material in context. Netherlands: Springer; 2007. doi:10.1007/0-306-48385-8.
[2] Ghasemi MR, Ehsani A. A hybrid radial-based Neuro-GA multiobjective design of laminated composite plates under moisture and thermal actions, inter. J Comput Inform Syst Sci Eng 2007;2(1):109-16.

[3] Hansel W, Treptow A, Becker W, Freisleben B. A heuristic and a genetic topology optimization algorithm for weight-minimal laminate structures. Compos Struct 2002;58(2):287-94. doi:10.1016/J.COMPSTRUCT.2009.09.039.

[4] Pohlak M, Majak J, Karjust K, Kuttner R. Multi-criteria optimization of large composite parts. Compos Struct 2010;92(9):2146-52. doi:10.1016 I.COMPSTRUCT.2009.09.039.

[5] Sebaey TA, Lopes CS, Blanco N, Costa J. Ant colony optimization for dispersed laminated composite panels under biaxial loading. Compos Struct 2011;94(1):31-6. doi:10.1016/J.COMPSTRUCT.2011.07.021.

[6] Deb K. Multi-objective optimisation using evolutionary algorithms. Wiley; 2003.

[7] Deb K, Agrawal S, Pratap A, Meyarivan T. A fast and elitist multi-objective genetic algorithm: NSGA-II. IEEE Trans Evolut Comput 2002;6(2):182-97.

[8] Gen M, Cheng R. Genetic algorithm and engineering design. 1st ed. New York: John Wiley and Sons, Inc.; 1997.

[9] Oller S. Simulacion numerica del comportamiento mecanico de los materiales compuestos. Barcelona: CIMNE; 2003.

[10] Kenndedy J, Eberhart R. Particle swarm optimization. In: Proceeding of IEEE international conference on neural networks, vol. IV; 1995. p. 1942-8.

[11] Lee DS, Bugeda G, Periaux J, Onate E. Robust active shock control bump design optimisation using parallel hybrid-MOGA. In: The 23rd international conference on parallel computational fluid dynamics 211, Barcelona, Spain 16-20 May: 2011.

[12] Lee DS, Periaux J, Onate E, Gonzalez LF. Advanced computational intelligence system for inverse aeronautical design optimisation. In: The 2011 Future Technology Research Association International (FTRA) - Internationa Conference on Advanced Software Engineering (ICASE-11), Busan, Korea, 2628 May; 2011

[13] Truesdell C, Toupin R. The classical field theories, Berlín; 1960

[14] Rastellini F, Oller S, Salomón O, Oñate E. Composite material non-linear modelling for long fibre-reinforced laminates. Continuum basis, computational aspects and validations. Comput Struct 2008;86:879-96.

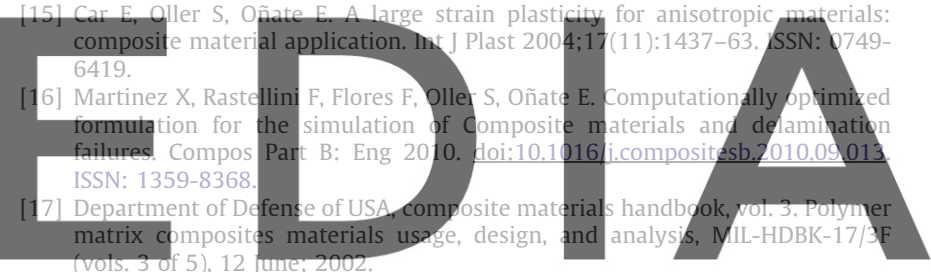

